\title{
Utilizing Fast Spin Echo MRI to Reduce Image Artifacts and Improve Implant/Tissue Interface Detection in Refractory Parkinson's Patients with Deep Brain Stimulators
}

\author{
Subhendra N. Sarkar, ${ }^{1}$ Pooja R. Sarkar, ${ }^{2}$ Efstathios Papavassiliou, ${ }^{3}$ and Rafael R. Rojas ${ }^{1}$ \\ ${ }^{1}$ Department of Radiology, Beth Israel Deaconess Medical Center, Harvard Medical School, 330 Brookline Avenue, \\ Boston, MA 02215, USA \\ ${ }^{2}$ School of Medicine, University of Texas Health Sciences Center, San Antonio, TX 78229, USA \\ ${ }^{3}$ Division of Neurosurgery, Beth Israel Deaconess Medical Center, Harvard Medical School, 330 Brookline Avenue, \\ Boston, MA 02215, USA
}

Correspondence should be addressed to Subhendra N. Sarkar; ssarkar@bidmc.harvard.edu

Received 31 October 2013; Accepted 5 January 2014; Published 25 February 2014

Academic Editor: Heinz Reichmann

Copyright ( 2014 Subhendra N. Sarkar et al. This is an open access article distributed under the Creative Commons Attribution License, which permits unrestricted use, distribution, and reproduction in any medium, provided the original work is properly cited.

\begin{abstract}
Introduction. In medically refractory Parkinson's disease (PD) deep-brain stimulation (DBS) is an effective therapeutic tool. Postimplantation MRI is important in assessing tissue damage and DBS lead placement accuracy. We wanted to identify which MRI sequence can detect DBS leads with smallest artifactual signal void, allowing better tissue/electrode edge conspicuity. Methods. Using an IRB approved protocol 8 advanced PD patients were imaged within MR conditional safety guidelines at low RF power (SAR $\leq 0.1 \mathrm{~W} / \mathrm{kg}$ ) in coronal plane at $1.5 \mathrm{~T}$ by various sequences. The image slices were subjectively evaluated for diagnostic quality and the lead contact diameters were compared to identify a sequence least affected by metallic leads. Results and Discussion. Spin echo and fast spin echo based low SAR sequences provided acceptable image quality with comparable image blooming (enlargement) of stimulator leads. The mean lead diameters were $2.2 \pm 0.1 \mathrm{~mm}$ for $2 \mathrm{D}, 2.1 \pm 0.1 \mathrm{~mm}$ for $3 \mathrm{D}$, and $4.0 \pm 0.2 \mathrm{~mm}$ for $3 \mathrm{D}$ MPRAGE sequence. Conclusion. Low RF power spin echo and fast spin echo based 2D and 3D FSE sequences provide acceptable image quality adjacent to DBS leads. The smallest artifactual blooming of stimulator leads is present on 3D FSE while the largest signal void appears in the 3D MPRAGE sequence.
\end{abstract}

\section{Introduction}

In medically refractory Parkinson's disease, deep brain stimulation (DBS) is often an effective therapeutic tool as noted by Obeso et al. [1] acting on the cells and fibers located closest to the implanted electrode. However, it remains unclear exactly how DBS therapy improves symptoms in Parkinson's patients, and the benefits of DBS are currently understood only empirically Okun [2]. Around the lead track, a thin capsule of connective tissue (thickness 5 to $25 \mu \mathrm{m}$ ) is formed surrounded by a $500 \mu \mathrm{m}$ or less rim of fibrillary gliosis as noted by Haberler et al. [3] after postmortem examination. In the adjacent brain tissue of thickness less than $1 \mathrm{~mm}$, loosely scattered glial fibrillary acidic protein-positive protein astrocytes are found and stimulation seems to modify the tissue microstructure of the local encapsulation, increasing conductivity and decreasing electrode impedance. It is important to directly visualize or indirectly estimate the size and relative contrast of surrounding tissue, preferably by MRI.

The goals of this study were twofold: first we wanted to see if MRI detectable leads have large size difference for patients immediately after implantation with no observable complications, and, second, we sought to explore if there are MR sequence specific size differences in visualized signal voids for DBS leads. The role of MRI for assessing DBS lead placement accuracy has already demonstrated [4] significant advantages despite ignoring the lead-tissue interface.

DBS leads are built with conducting metals ( $\mathrm{Pt} / \mathrm{Ir}$ alloy) that are mildly paramagnetic and produce artifactual signal 
loss in vivo for all MRI sequences. We asked the questions: what the limit of edge detection and localizability of DBS leads is, and which sequence minimizes the blooming artifacts. In other words, it is important to improve visualization of tissue edges adjacent to implant tips and minimize tissue signal loss due to high susceptibility of metallic components in the implanted electrodes.

During an MRI the DBS electrodes deposit a significant amount of radiofrequency induced heat due to the metallic composition (represented by specific absorption rate, SAR) to the surrounding tissue as described by Zrinzo et al. [5] and Tagliati et al. [6]. Hence the DBS vendor Medtronic Inc. and the FDA have provided MR conditional guidance $[7,8]$ for imaging hardware and a maximum SAR level allowed for imaging of patients with DBS. The present work has followed these restrictions during the development and testing phases on non-DBS subjects and the results have been reported elsewhere by Sarkar et al. [9].

\section{Materials and Methods}

2.1. Patient Selection. Following institutional ethics and research review committee guidelines at our institution, eight patients with advanced Parkinson's disease that were refractory to medications were studied after implantation of DBS for lead localization (4 males, 4 females, age range 5375 years, disease duration $8-16$ years, median 11.5 years, and UPDRS score: more than 30 without medication; medical history included dyskinesia, speech and/or memory difficulty, depression, and gait disorder).

2.2. Imaging Details. MR sequences used were $2 \mathrm{D}$ fast spin echo T2 (FSE T2) for 4 patients, 3D FSE T2 for 2 patients, Spin echo T1 (SE T1) for 2 patients, and magnetization prepared rapid acquisition gradient echo T1 (MPRAGE T1) for all 8 patients. However, for DBS patients the sequences were prepared so as to run at a low RF power (at SAR < $0.1 \mathrm{~W} / \mathrm{kg}$ ). Following earlier reports [9], we have stretched the refocusing RF pulses by $2-4$-fold and reduced the refocusing angles substantially to attain a whole head RF power level of $0.1 \mathrm{~W} / \mathrm{kg}$ as required per vendor and FDA guidelines [7, 8]. Two readers (EP and RR, both with more than 10 years of clinical experience) judged the imaging efficacy and clinical quality of low SAR images.

In addition to the T2 sequences, two other sequences with spin echo origin (FLAIR T2 and SE T1) were used at low SAR. The FLAIR sequence was qualitatively assessed for infection or other indications and was not used for DBS lead size measurements. Traditionally an inversion-prepared GRE-based high resolution sequence (MPRAGE) is used to image gray/white matter and the DBS leads at a high resolution (typically $1 \times 1 \times 1 \mathrm{~mm}^{3}$ isotropic or $1 \times 1 \times 1.5 \mathrm{~mm}^{3}$ voxel resolution). Being a gradient echo based method, this sequence is sensitive to susceptibility and causes a greater amount of signal loss surrounding the metallic leads. We used the lead diameter from MPRAGE as the maximum artifact size that may be acceptable for visualizing adjacent gray or white matter.
2.3. Image-Based Measurements and Statistical Analysis. Each of the DBS leads has 4 contacts $(1.5 \pm 0.1 \mathrm{~mm}$ in height and $1.3 \pm 0.1 \mathrm{~mm}$ in diameter, separated by plastic sheaths). The diameters of one or more contacts in their largest dimensions were measured from magnified MR images as shown in Figure 1(c). A consistent radiologic intensity window/level was used for all measurements by a single reader, SS. From all 8 patients, one estimate of lead diameter per patient was measured for MPRAGE and was averaged to obtain a mean $D_{\text {MPRAGE }}$. From four patients scanned with 2D FSE T2 sequence, two lead contact estimates per patient were measured and a total of 8 estimates were averaged to arrive at a mean $D_{2 \mathrm{D} \text { FSE T2 }}$. Four lead contact estimates per patient for each of two patients with 3D FSE T2 sequences, a total of 8 estimates, were averaged and a mean $D_{3 \mathrm{D} \text { FSE T2 }}$ was obtained. Finally, four lead contact estimates per patient for two patients with $2 \mathrm{D}$ spin echo $\mathrm{T} 1$ results, a total of 8 estimates were averaged and a mean $D_{2 \mathrm{D} \mathrm{SE} \mathrm{T1}}$ was obtained. Six null hypotheses were drawn to test pairwise equivalence of means from the four sequences. A nonparametric statistical test (two-tailed Wilcoxon signed-rank test) was used to draw conclusions about the mean diameter differences at a significance level of 0.05 (Table 1).

\section{Results}

In Figures 1 and 2 typical coronal images from all 4 sequences are shown. In addition the low TE (proton density, Figure 1(c)) and long TE version of 2D FSE (Figure 1(d)) are also compared.

Both the readers (EP, RR) concluded that although the low SAR images are somewhat grainy (due to lower signal-tonoise) cerebral tissue conspicuity away from as well as adjacent to the DBS leads was adequate for radiologic diagnosis with no noticeable quality difference among spin echo, fast spin echo, and MPRAGE sequences. As shown in Figure 2, the FLAIR version of low SAR FSE sequence with long echo train (80-100) and low refocusing flip angles produces similar DBS appearance and is useful for imaging of infections and other complications.

The measured mean lead diameters from the MR images were as follows:

$$
\begin{aligned}
& D_{\text {MPRAGE }}=4.0 \pm 0.2 \mathrm{~mm}, \\
& D_{\text {2D SE T1 }}=2.2 \pm 0.1 \mathrm{~mm}, \\
& D_{\text {2D FSE T2 }}=2.2 \pm 0.1 \mathrm{~mm}, \\
& D_{3 \text { D FSE T2 }}=2.1 \pm 0.1 \mathrm{~mm} .
\end{aligned}
$$

The range of lead contact sizes measured from images for all 8 estimates is listed in Table 1 . The mean values represent the limit attainable for each MR sequence with long echo train 3D FSE T2 being the best. A $1.3 \mathrm{~mm}$ standard DBS lead contact including perhaps a $0.5 \mathrm{~mm}$ encapsulation layer is visualized with the least artifactual signal void of $2.1 \mathrm{~mm}$ when 3D FSE T2 MR sequence is used. Two-dimensional FSE $\mathrm{T} 2$ at long TE $(85 \mathrm{~ms})$ or SE T1 at short TE $(14 \mathrm{~ms})$ is also able to restrict the artifactual blooming of DBS contacts and encapsulation layers to a total of $2.2 \mathrm{~mm}$ size signal void while 


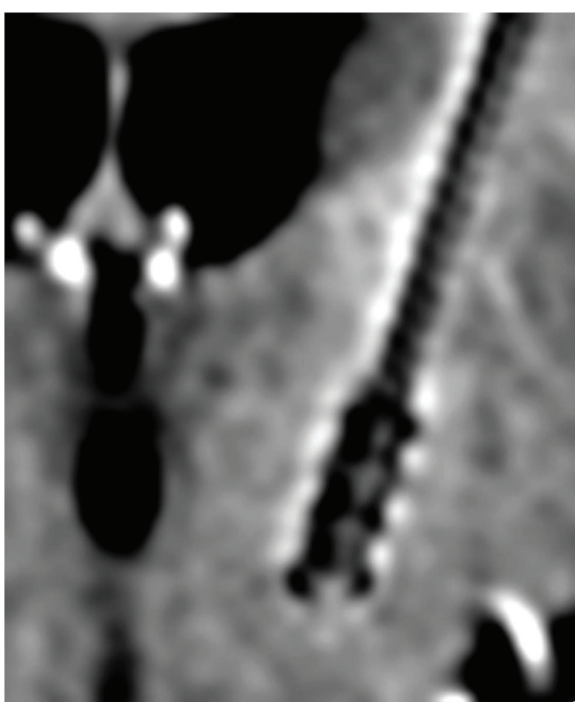

(a)

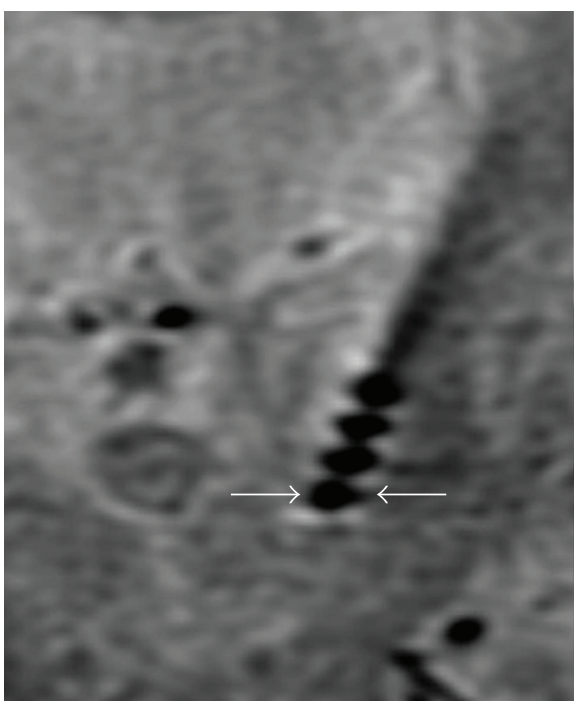

(c)

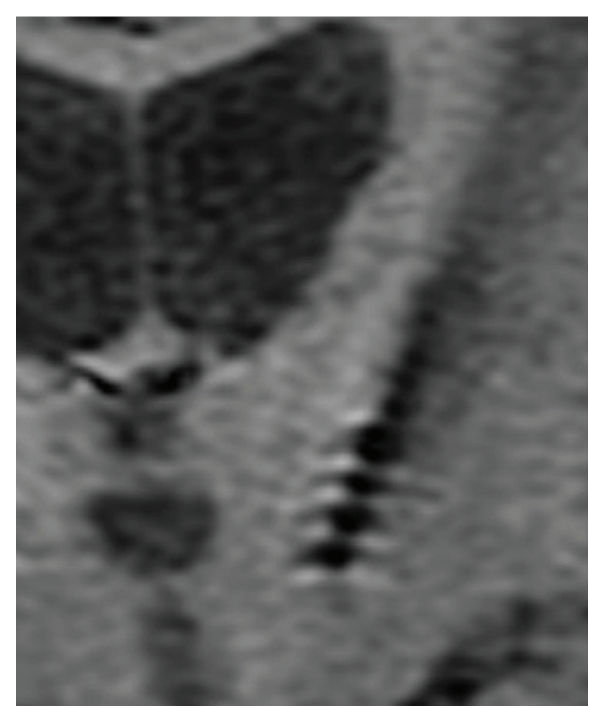

(b)

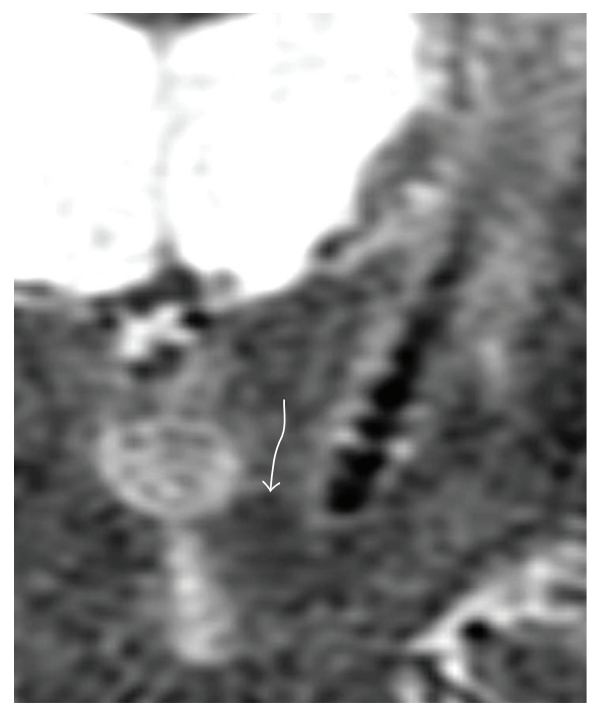

(d)

Figure 1: Typical coronal MR images, (a) a section from 3D MPRAGE; measured contact diameter (measurement plan is shown in panel (c) by straight arrows) $=4 \mathrm{~mm}$, more than that from (b) a section from 2D SE T1 (2.2 mm) or (c) 2D FSE PD (2.3 mm) and (d) 2D FSE T2 $(2.3 \mathrm{~mm})$ sequences. The location of the lead at left subthalamic nucleus is indirectly estimated as $11-12 \mathrm{~mm}$ lateral to midline across the superior-anterior border of the red nucleus (curved arrow, panel (d)).

TABLE 1: The range of lead contact measurements from various MR sequences.

\begin{tabular}{|c|c|c|c|c|}
\hline Lead tip-size measurements & $D_{\text {MPRAGE }}(\mathrm{mm})$ & $D_{\text {2D SE Tl }}(\mathrm{mm})$ & $D_{\text {2D FSE T2 }}(\mathrm{mm})$ & $D_{3 \text { DFSET2 }}(\mathrm{mm})$ \\
\hline 1 & 3.8 & 2.1 & 2.3 & 2.2 \\
\hline 2 & 3.9 & 2.3 & 2.1 & 2.2 \\
\hline 3 & 4.1 & 2 & 2.2 & 2.1 \\
\hline 4 & 4.2 & 2.2 & 2 & 2.1 \\
\hline 5 & 3.9 & 2.2 & 2.1 & 2 \\
\hline 6 & 3.8 & 2.2 & 2.1 & 2 \\
\hline 7 & 3.7 & 2.1 & 2.2 & 2 \\
\hline 8 & 4.3 & 2.2 & 2.3 & 2.1 \\
\hline Mean diameter $(\mathrm{mm})$ & $4 \pm 0.2$ & $2.2 \pm 0.1$ & $2.2 \pm 0.1$ & $2.1 \pm 0.1$ \\
\hline
\end{tabular}




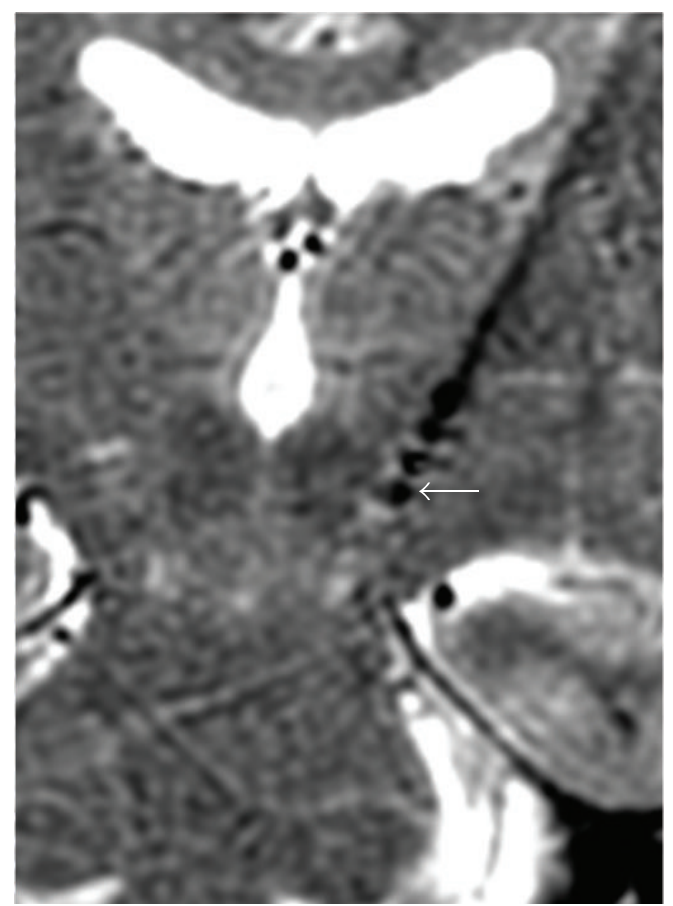

(a)

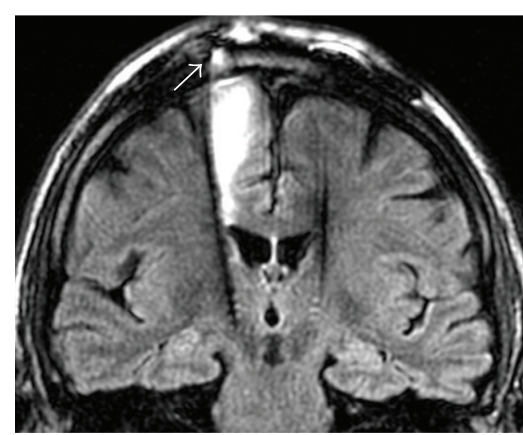

(b)

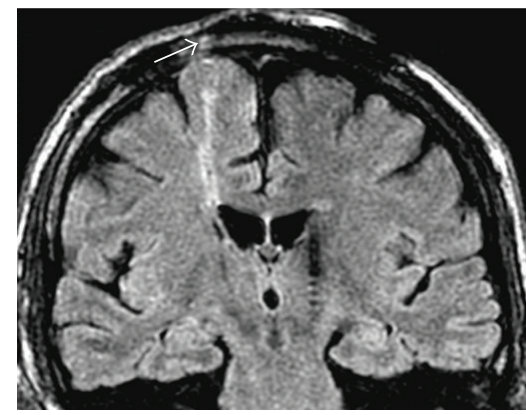

(c)

FIgURE 2: (a) Coronal 3D long echo train T2 image showing $2.2 \mathrm{~mm}$ tip diameter (ETL 80, TE $85 \mathrm{~ms}$ ) with visualization of white matter tissue adequately; (b) and (c) 3D long echo train FLAIR MR signal for an infected DBS lead (arrow showing infection prior to removal (b) and after removal and treatment (c)). Note the artifactual bright dots in T2 images are not prominently present on FLAIR while the size of the implant lead is similar to those in T2 images (Figure 1).

TABLE 2: Wilcoxon signed-rank test results for visualized DBS lead diameters from images using various MR sequences for DBS patients (C.I. $\alpha=0.05$ ).

\begin{tabular}{|c|c|c|c|}
\hline Test number & Null hypotheses mean values (mm) & $W, W_{\text {crit }}, P$ value & Test results \\
\hline I. & $\begin{array}{c}H_{0}:\left(D_{\text {MPRAGE }}-D_{2 \mathrm{D} \mathrm{SET1}}\right)=0 \\
4.0 \pm 0.2 ; 2.2 \pm 0.1\end{array}$ & $0,3, P \leq 0.05$ & Reject $H_{0}$ (diameters are significantly different) \\
\hline II. & $\begin{array}{c}H_{0}:\left(D_{\text {MPRAGE }}-D_{\text {3D FSE T2 }}\right)=0 \\
4.0 \pm 0.2 ; 2.1 \pm 0.1\end{array}$ & $0,3, P \leq 0.05$ & Reject $H_{0}$ (diameters are significantly different) \\
\hline III. & $\begin{array}{c}H_{0}:\left(D_{\text {MPRAGE }}-D_{\text {2D FSE T2 }}\right)=0 \\
4.0 \pm 0.2 ; 2.2 \pm 0.1\end{array}$ & $0,3, P \leq 0.05$ & Reject $H_{0}$ (diameters are significantly different) \\
\hline IV. & $\begin{array}{c}H_{0}:\left(D_{2 \mathrm{D} \mathrm{FSE} \mathrm{T} 2}-D_{2 \mathrm{D} \mathrm{SE} \mathrm{T1}}\right)=0 \\
2.1 \pm 0.1 ; 2.2 \pm 0.1\end{array}$ & $17,3, P \leq 0.05$ & Accept $H_{0}$ (diameters are not significantly different) \\
\hline V. & $\begin{array}{c}H_{0}:\left(D_{3 \text { D FSE T2 }}-D_{2 \text { D SE T1 }}\right)=0 \\
2.1 \pm 0.1 ; 2.2 \pm 0.1\end{array}$ & $8,3, P \leq 0.05$ & Accept $H_{0}$ (diameters are not significantly different) \\
\hline VI. & $\begin{array}{c}H_{0}:\left(D_{3 \text { D FSE T2 }}-D_{\text {2D FSE T2 }}\right)=0 \\
2.1 \pm 0.1 ; 2.2 \pm 0.1\end{array}$ & $8,3, P \leq 0.05$ & Accept $H_{0}$ (diameters are not significantly different) \\
\hline
\end{tabular}

short TE (2.3 ms) MPRAGE produces the most artifactual signal loss ( $4 \mathrm{~mm}$ on an average). At longer TE values (T2* weighted gradient echo) the artifacts bloom beyond acceptability for diagnostic use and sequences including susceptibility weighted imaging (SWI) were not tested on DBS recipients.

Note that the range of measured diameters is approximately $\pm 5 \%$ of the mean values for all MR sequences. The standard dimensions of the cylindrical Medtronic 3387/3389 DBS electrode contacts are specified as $1.5 \pm 0.1 \mathrm{~mm}$ height by $1.3 \pm 0.1 \mathrm{~mm}$ diameter. The electrode encapsulation layer thickness is $0.5 \pm 0.4 \mathrm{~mm}$, Haberler et al. [3], Moss et al. [10].

The pairwise statistical mean difference test results are noted in Table 2 and essentially indicate equivalent blooming results for all the $2 \mathrm{D}$ sequences while suggesting $3 \mathrm{D}$ FSE as the best and 3D MPRAGE as the worst as far as the visualized 
DBS diameters are concerned. The critical limit attainable to contain the artifactual lead size by any sequence seems to be approximately $2.1 \pm 0.1 \mathrm{~mm}$.

\section{Discussion}

To our knowledge, this is the first MR work that utilizes very low RF power on refractory Parkinson's patients to study the lead contact size in vivo and relates it to the MRI sequences. The results demonstrate that by lowering echo time or increasing echo train lengths in fast spin echo the tissue visualization surrounding the DBS contacts is better than that obtainable by gradient echo based imaging and it is possible to achieve a fairly small metallic artifact blooming from the DBS leads.

In this work, we assumed the electrode surface is perfectly smooth and ignore any electrode corrosion and surface modification that may occur as a result of implantation process or early stages of stimulation treatment. However, analysis of postmortem or explanted DBS electrodes does not show any visible surface modification of the metallic contacts or tissue changes around the active contact and nonstimulated areas adjacent to the insulated parts as reported by Haberler et al. [3] and Moss et al. [10].

One may expect that the chosen sequence that performs better for lead blooming and tissue contrast would use perfect refocusing RF pulses, long echo train length (ETL), and short echo time (TE). However, in Figure 1, we notice that there is a limit, approximately $2.2 \mathrm{~mm}$ diameter of lead size, that is reached even with the minimum TE or using a perfect $180^{\circ}$ refocusing condition (as in spin echo $\mathrm{T} 1$ ). The artifact is not reduced when echo train is long, as in routine $2 \mathrm{D}$ FSE T2 with 16 echo trains. The use of a very long echo train (80-100) as in 3D FSE T2 MR sequence, even without using perfect $180^{\circ}$ refocusing conditions as developed earlier for non-DBS subjects by Sarkar et al. [9], performs the best among the 4 sequences tested. Note that these 3D FSE sequences use optimal, much lower refocusing pulse angles than $180^{\circ}$. One may speculate, based on these results, that the current imaging limit to attain minimum artifactual blooming for MR imaging for a $1.3 \mathrm{~mm}$ diameter standard DBS lead contacts surrounded by perhaps a $0.5 \mathrm{~mm}$ encapsulation layer is approximately $2.1 \mathrm{~mm}$.

Currently, a number of groups use MRI for assessing DBS lead placement accuracy using high SAR [4] or at ultralow SAR [11, 12]. Three-dimensional MPRAGE is often used to assess the lead placement without directly visualizing the deep brain nuclei that are more conspicuous by FSE sequences. This work shows that for DBS localization there are advantages for using $3 \mathrm{D}$ acquisition. With $2 \mathrm{D}$ imaging sequences, the slice profiles are less perfect than $3 \mathrm{D}$ and often a slice gap is needed to avoid interslice signal contamination. Hence 3D sections are expected to perform better than the 2D counterparts of similar thickness for lead position verification and assessment of adjacent tissue viability in case of suspected complications.

4.1. Limitations of the Study. The tissue edge visualization depends on the MR signal and image resolution which directly depend on the magnet field strength, quality of RF coils, and the clinically feasible imaging time. For safety reasons, Parkinson's patients with DBS can currently be imaged only at $1.5 \mathrm{~T}$ and with transmit-receive local head coils. Each of these hardware components operates at approximately half the performance level compared to those available for routine patients. Hence refractory PD patients are being imaged at 4 -fold lower imaging sensitivity than those imaged at $3 \mathrm{~T}$ with multielement head array coils. In addition, due to RF power deposition issues, all of our imaging in this work was done at very low SAR level $(0.1 \mathrm{~W} / \mathrm{kg})$ that also limits, MR signal-to-noise and extends imaging time. Therefore, with improved hardware and RF safety limits, our results may improve and one may be able to use impedance models and related developments [13] to image substructures around the implant leads in near future more accurately than obtained in this work. Finally, our sample size should be extended to a larger patient population including patients after longer DBS treatments to image the role of and treatment effects on encapsulation layer.

\section{Conclusions}

Conforming to safe RF power limits, this is the first work reporting MRI sequence dependent artifact size of DBS leads in refractory Parkinson's patients and exploring various sequence limits to minimize the artifactual signal void within clinically feasible image resolution and hardware limitations for PD patients. Spin echo and fast spin echo based 2D and 3D FSE sequences provide comparable image blooming of stimulator leads while 3D FSE produces the smallest artifact and the gradient echo based 3D MPRAGE produces the largest.

\section{Abbreviations \\ PD: Parkinson's disease \\ SAR: Specific absorption rate \\ DBS: Deep brain stimulator or stimulation \\ SE: Spin echo \\ FSE: Fast spin echo.}

\section{Disclosure}

None of the authors have any direct or indirect financial conflict of interests to disclose.

\section{Conflict of Interests}

The authors declare that there is no conflict of interests regarding the publication of this paper.

\section{Authors' Contribution}

Subhendra N. Sarkar contributed to the conception and execution of the research project and also wrote the first draft of the paper. Pooja R. Sarkar was responsible for the hypothesis design and statistical analysis as well as manuscript editing 
while Efstathios Papavassiliou contributed to the execution of the research project and the review and critique of the paper. Rafael Rojas organized the research project and also participated in the review and critique of the paper.

\section{Acknowledgment}

The authors would like to acknowledge the help from Reed F. Busse, Applied Science Laboratory, GE Healthcare, Madison, WI, for providing them with an early version of 3D FSE sequence and related discussions during the year 2009 for sequence optimization.

\section{References}

[1] J. A. Obeso, C. W. Olanow, M. C. Rodriguez-Oroz, P. Krack, R. Kumar, and A. E. Lang, "Deep-brain stimulation of the subthalamic nucleus or the pars interna of the globus pallidus in Parkinson's disease," The New England Journal of Medicine, vol. 345, no. 13, pp. 956-963, 2001.

[2] M. S. Okun, "Deep-brain stimulation for Parkinson's disease," The New England Journal of Medicine, vol. 367, pp. 1529-1538, 2012.

[3] C. Haberler, F. Alesch, P. R. Mazal et al., "No tissue damage by chronic deep brain stimulation in Parkinson's disease," Annals of Neurology, vol. 48, pp. 372-376, 2000.

[4] P. S. Larson, R. M. Richardson, P. A. Starr, and A. J. Martin, "Magnetic resonance imaging of implanted deep brain stimulators: experience in a large series," Stereotactic and Functional Neurosurgery, vol. 86, no. 2, pp. 92-100, 2008.

[5] L. Zrinzo, F. Yoshida, M. I. Hariz et al., "Clinical safety of brain magnetic resonance imaging with implanted deep brain stimulation hardware: large case series and review of the literature," World Neurosurgery, vol. 76, no. 1-2, pp. 164-172, 2011.

[6] M. Tagliati, J. Jankovic, F. Pagan, F. Susatia, I. U. Isaias, and M. S. Okun, "Safety of MRI in patients with implanted deep brain stimulation devices," NeuroImage, vol. 47, no. 2, pp. T53-T57, 2009.

[7] Medtronic: MRI Guidelines For Medtronic Deep Brain Stimulation Systems, Medtronic Inc., Minneapolis, Minn, USA, 2007, http://professional.medtronic.com/wcm/groups/mdtcom_sg/@ mdt/@neuro/documents/documents/dbs-2007-mri.pdf.

[8] T. Woods, Establishing Safety and Compatibility of Passive Implants in the Magnetic Resonance (MR) Environment. Guidance for Industry and FDA Staff, Center for Devices and Radiological Health, FDA, Silver Springs, Md, USA, 2008.

[9] S. N. Sarkar, D. C. Alsop, A. J. Madhuranthakam et al., "Brain MR imaging at ultra-low radiofrequency power," Radiology, vol. 259, no. 2, pp. 550-557, 2011.

[10] J. Moss, T. Ryder, T. Z. Aziz, M. B. Graeber, and P. G. Bain, "Electron microscopy of tissue adherent to explanted electrodes in dystonia and Parkinson's disease," Brain, vol. 127, no. 12, pp. 2755-2763, 2004.

[11] S. N. Sarkar, E. Papavassiliou, R. Rojas et al., "Cerebral tissue contrast is preserved even at very low radio frequency MRI power for inversion recovery imaging of Parkinson's patients with deep brain stimulators," American Journal of Neuroradiology, vol. 35, p. 30, 2014.
[12] S. N. Sarkar, E. Papavassiliou, D. B. Hackney et al., "Threedimensional brain MRI for DBS patients within ultra-low radiofrequency power limits," Movement Disorders, 2014.

[13] C. R. Butson, C. B. Maks, and C. C. McIntyre, "Sources and effects of electrode impedance during deep brain stimulation," Clinical Neurophysiology, vol. 117, no. 2, pp. 447-454, 2006. 


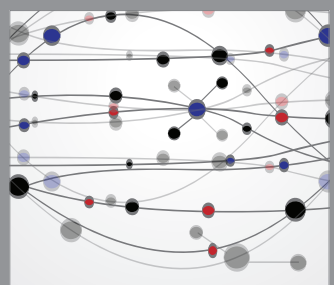

The Scientific World Journal
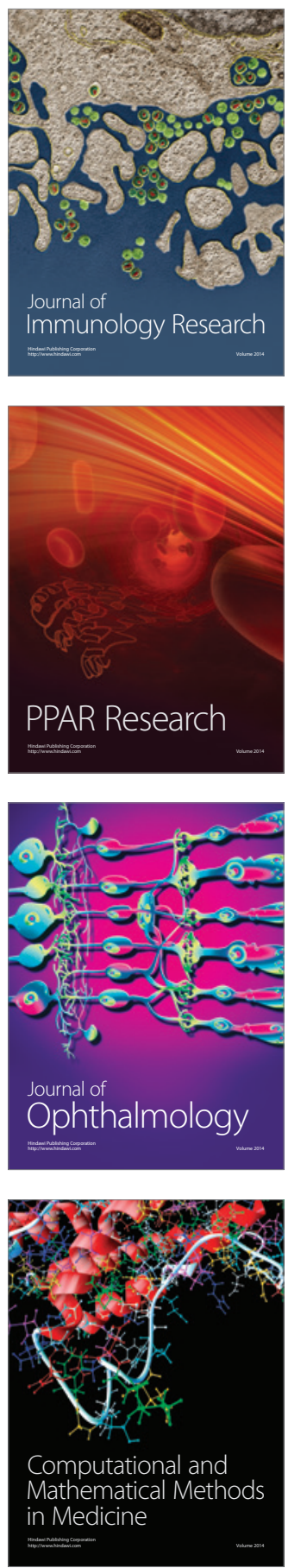

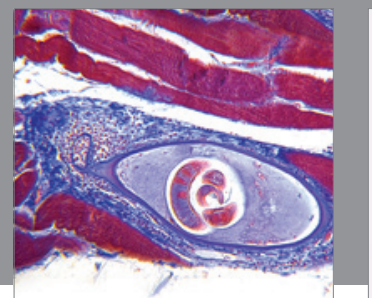

Gastroenterology

Research and Practice
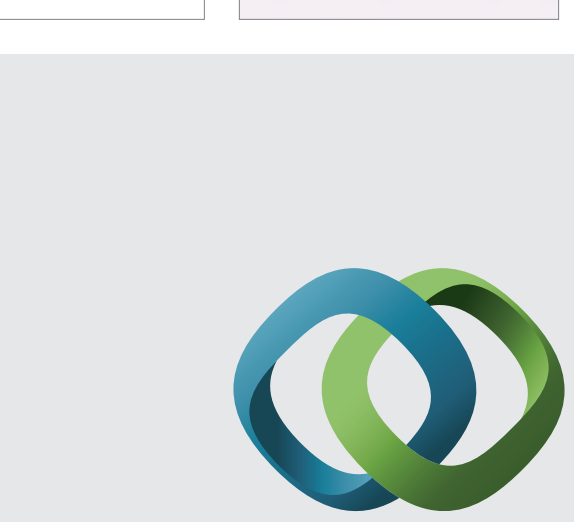

\section{Hindawi}

Submit your manuscripts at

http://www.hindawi.com
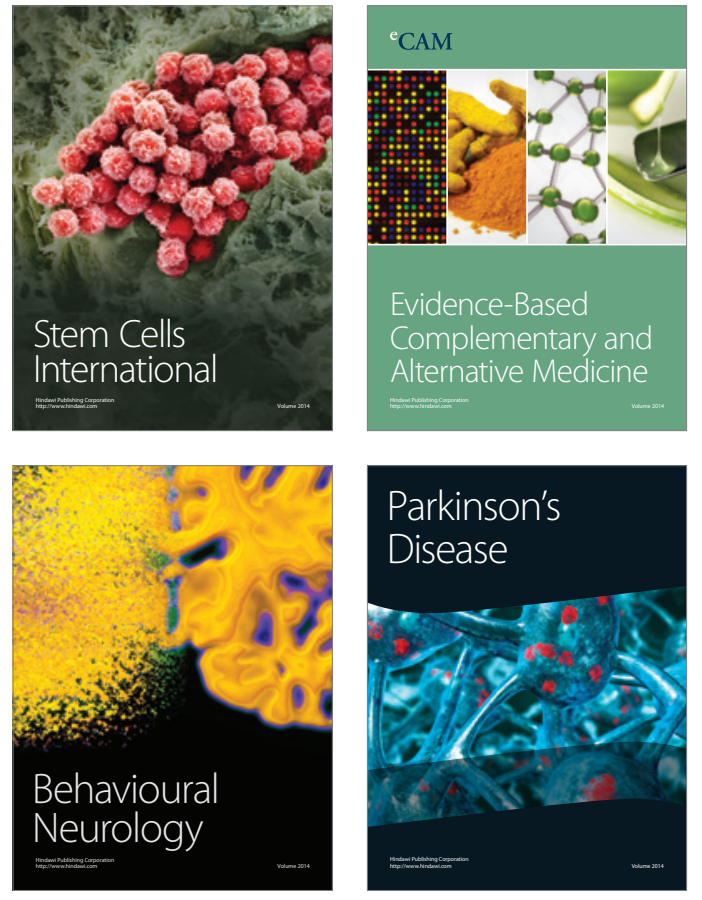
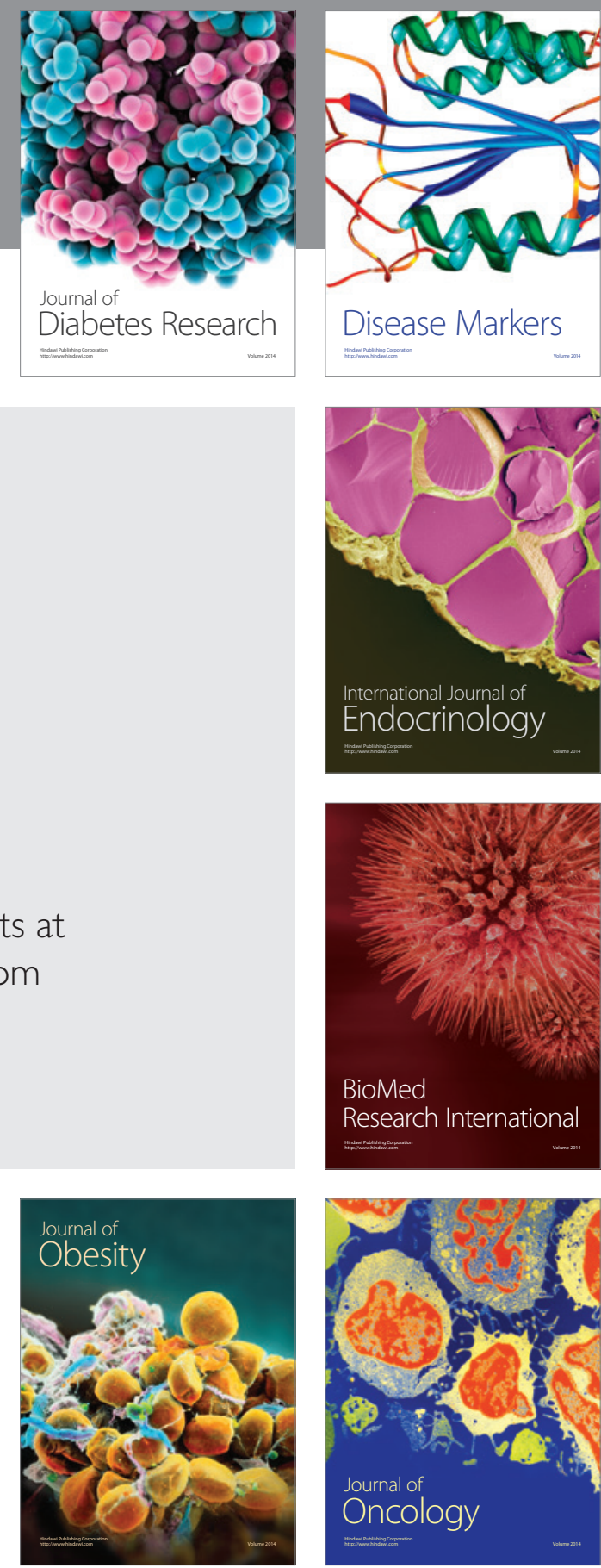

Disease Markers
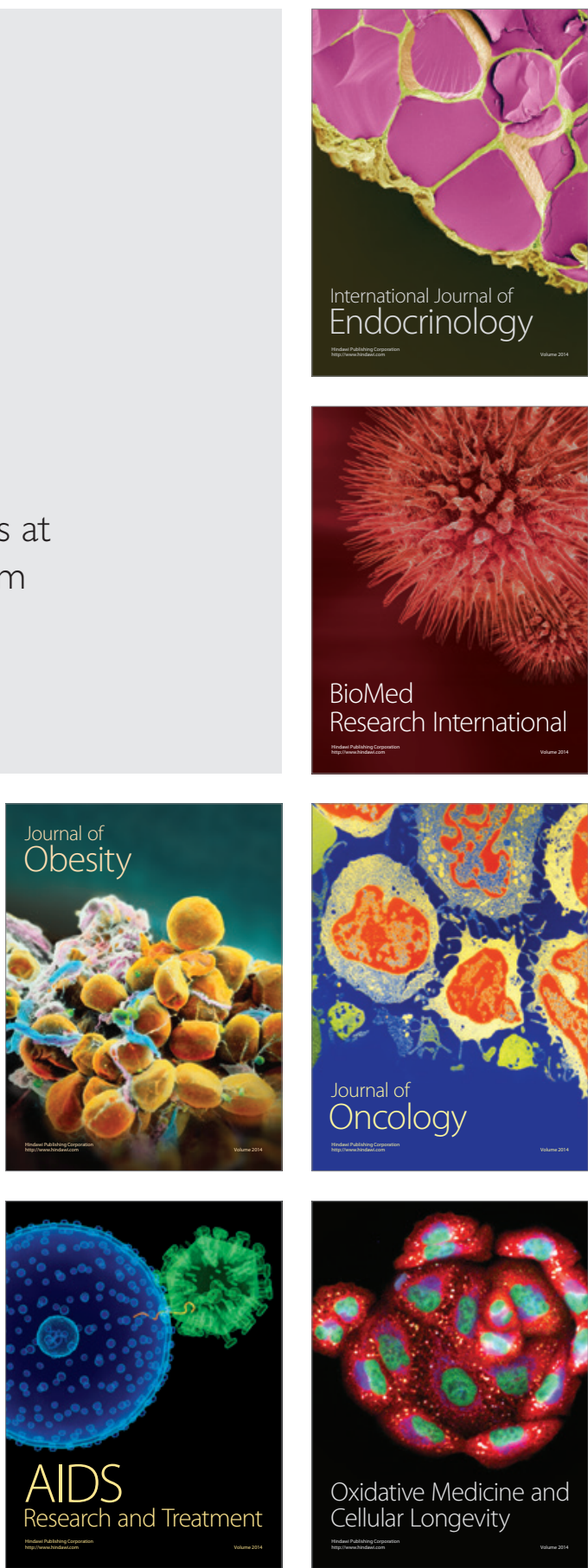